\title{
Comparison of hepatic MDCT, MRI, and DSA to explant pathology for the detection and treatment planning of hepatocellular carcinoma
}

\author{
Lauren M. Ladd, Temel Tirkes, Mark Tann, David M. Agarwal, Matthew S. Johnson, Bilal Tahir, and \\ Kumaresan Sandrasegaran \\ Department of Radiology and Imaging Sciences, Indiana University School of Medicine, Indianapolis, IN, USA
}

Background/Aims: The diagnosis and treatment plan for hepatocellular carcinoma (HCC) can be made from radiologic imaging. However, lesion detection may vary depending on the imaging modality. This study aims to evaluate the sensitivities of hepatic multidetector computed tomography (MDCT), magnetic resonance imaging (MRI), and digital subtraction angiography (DSA) in the detection of HCC and the consequent management impact on potential liver transplant patients.

Methods: One hundred and sixteen HCC lesions were analyzed in 41 patients who received an orthotopic liver transplant (OLT). All of the patients underwent pretransplantation hepatic DSA, MDCT, and/or MRI. The imaging results were independently reviewed retrospectively in a blinded fashion by two interventional and two abdominal radiologists. The liver explant pathology was used as the gold standard for assessing each imaging modality.

Results: The sensitivity for overall HCC detection was higher for cross-sectional imaging using MRI $51.5 \%, 95 \%$ confidence interval $[\mathrm{Cl}]=36.2-58.4 \%)$ and $\mathrm{MDCT}(49.8 \%, 95 \% \mathrm{Cl}=43.7-55.9 \%)$ than for DSA (41.7\%, 95\% Cl=36.2-47.3\%) $(P=0.05)$. The difference in false-positive rate was not statistically significant between MRI $(22 \%)$, MDCT $(29 \%)$, and DSA (29\%) $(P=0.67)$. The sensitivity was significantly higher for detecting right lobe lesions than left lobe lesions for all modalities (MRI: $56.1 \%$ vs. $43.1 \%$, MDCT: $55.0 \%$ vs. $42.0 \%$, and DSA: $46.9 \%$ vs. $33.9 \%$; all $P<0.01$ ). The sensitivities of the three imaging modalities were also higher for lesions $\geq 2 \mathrm{~cm}$ vs. $<2 \mathrm{~cm}$ (MRI: $73.4 \%$ vs. $32.7 \%$, MDCT: $66.9 \%$ vs. $33.8 \%$, and DSA: $62.2 \%$ vs. $24.1 \%$; all $P<0.01$ ). The interobserver correlation was rated as very good to excellent.

Conclusions: The sensitivity for detecting HCC is higher for MRI and MDCT than for DSA, and so cross-sectional imaging modalities should be used to evaluate OLT candidacy. (Clin Mol Hepatol 2016;22:450-457)

Keywords: Hepatocellular carcinoma; Computed tomography; Magnetic resonance imaging; Digital subtraction angiography; Transplant

\section{INTRODUCTION}

Hepatocellular carcinoma (HCC) presents unique challenges and opportunities for patients and healthcare professionals globally. It is the 7th most common malignancy and 3rd leading cause of cancer death worldwide. ${ }^{1,2}$ In the United States, it ranks as the 5th

\section{Abbreviations:}

$\mathrm{Cl}$, confidence interval; DSA, digital subtraction angiography; HCC, hepatocellular carcinoma; MDCT, multidetector computed tomography; MRI, magnetic resonance imaging; OLT, orthotopic liver transpant

\section{Corresponding author: Temel Tirkes}

Department of Radiology and Imaging Sciences, Indiana University School of Medicine, $550 \mathrm{~N}$. University Blvd. Suite 0663, Indianapolis, IN, 46202, USA.

Tel: +1-317-944-8832, Fax: +1-317-944-1848

E-mail: atirkes@iupui.edu 
and 9th most common cause of cancer death in men and women, respectively. ${ }^{3}$ The incidence of HCC has continued to rise over the past several decades ${ }^{4,5}$ with approximately a $3 \%$ increase in incidence from 2004 to 2008 according to the American Cancer Society. ${ }^{3}$

Orthotopic liver transplant (OLT) eliminates the existing malignancy and the diseased liver, a source for future malignancies. Transplant recipients have a dramatic increase in 5-year survival rate, increasing to $84 \%$, compared to patients receiving localized treatment such as radiofrequency ablation (53\% at early stage), partial hepatectomy (47\%), and chemo- or radioembolization (35\%). ${ }^{6}$ However, the costs involved in this curative treatment are not trivial. In 2008, the estimated total cost for OLT from 30 days pre-transplant to 180 days post-transplant was $\$ 523,400$. $^{7}$ The financial burden, as well as limited availability of liver grafts, makes precise determination of transplant candidacy imperative and in many cases, imaging findings determine OLT candidacy. The radiologist not only makes the diagnosis of HCC without histological confirmation, ${ }^{8,9}$ but also is responsible for determining the number and size of HCC tumors that govern a patient's transplant candidacy versus a palliative pathway. ${ }^{8,10-13}$ Milan and UCSF criteria have been established for the purpose of most appropriate allocation of liver transplant resources. Both of these criteria necessitate the patient should have three or fewer HCCs in order to qualify for OLT. In 2008, the Organ Procurement and Transplant Network (OPTN) and United Network for Organ Sharing (UNOS) revised the imaging criteria guidelines for diagnosis of HCC due to the concern for a high number of false positives, which significantly impacts transplant qualification and allocation of valuable resources. ${ }^{14}$ As a result, smaller lesions $(1-2 \mathrm{~cm})$ must be confirmed histologically or monitored for a longer period of time before transplantation preference points are allocated.

Several studies have individually or comparatively evaluated the sensitivity for detecting HCCs by different imaging modalities; however, we have found no such study directly comparing sensitivity of HCC detection by multidetector computed tomography (MDCT), magnetic resonance imaging (MRI), and angiography with liver explant pathology correlation. There are also discrepancies in digital subtraction angiography (DSA) findings compared to pre-procedural cross sectional imaging, presenting a management dilemma. One recent study describes the detection of "additional lesions" during angiography not previously identified on MDCT and subsequent empiric treatment of these "lesions" with chemoembolization. ${ }^{15}$ If this approach is used, will DSA findings affect transplant candidacy? The purpose of this study was to compare the sensitivity of cross sectional imaging and hepatic an- giography to detect HCC based on the pathologic analysis of the explanted livers, and to investigate whether these imaging findings will help determine the most appropriate management and treatment pathway for these patients.

\section{MATERIALS AND METHODS}

\section{Patient selection criteria}

This study was Health Insurance Portability and Accountability Act (HIPAA)-compliant, and approved by the Institutional Review Board prior to study commencement. A total of 125 consecutive patients with HCC who underwent OLT over a 4-year period were reviewed. Forty-one subjects who met the following inclusion criteria were included: i) available MDCT and/or MRI images; ii) available hepatic angiography images; and iii) available liver explant pathology reports. The selection of transplant candidates was based on imaging diagnosis of HCC. ${ }^{11,16,17}$ Electronic medical records and picture archiving and communication system images of each patient were reviewed to identify and include the liver transplant patients with both hepatic angiography and cross sectional imaging (MDCT and/or MRI) performed prior to transplantation. Pretransplant hepatic angiograms were performed in all patients. Both MDCT and MRI were available for 31 patients, while the remaining 10 patients had only one form of cross sectional imaging available for review, including 8 with MDCT and 2 with MRI.

\section{MDCT}

Dual- or triple-phase contrast-enhanced MDCT exams were performed on 16-64 slice computed tomography scanners (Phillips Brilliance CT Scanner; Cleveland, OH, USA) with $120 \mathrm{kVp}$ and 242-496 mA depending on adjustments for patient size. Images were acquired at standard intervals of 25-35 seconds (arterial phase), 70-80 seconds (portal venous phase), and, in two patients, 5 minutes (delayed phase) after the administration of 100 $120 \mathrm{~mL}$ nonionic iodinated contrast (Isovue-370; Bracco Diagnostics Inc., Monroe Township, NJ, USA) via power injection (Medrad Stellant CT Injection Systems; Warrendale, PA, USA) at approximately $2.5-4 \mathrm{~mL} / \mathrm{second}$.

\section{MRI}

MRI was performed on 1.5 Tesla Magnetom Avanto magnetic 
resonance scanners (Siemens Medical Solutions, Malvern, PA, USA). Standard sequences included axial T1-weighted in- and outof-phase images, fat-suppressed axial T2-weighted images (HASTE: Siemens Medical Solutions, Malvern, PA, USA), coronal T2-weighted images (HASTE), and fat-suppressed axial T1 Volumetric Interpolated Breath-hold Examination (VIBE; Siemens Medical Solutions, Malvern, PA, USA) pre- and post-contrast images with a 320 x 168 matrix, 3 mm slice thickness, NEX=1, TR=4.61 $\mathrm{ms}, \mathrm{TE}=2.18 \mathrm{~ms}$. Dynamic post-contrast images were obtained in arterial, portal venous, and 5 minute delayed phases using sample bolus triggering method. The timing of arterial phase for dynamic post-contrast images was determined based on aortic peak sample bolus timing (18-20 seconds). A $15 \mathrm{~mL}$ gadolinium contrast bolus (gadobenate dimeglumine, Multihance ${ }^{\circledR}$, Bracco Diagnostics Inc., Monroe Township, NJ, USA) and $20 \mathrm{~mL}$ saline flush was administered via power injection (Spectris Solaris EP MR Injection System, Medrad, Warrendale, PA, USA).

\section{DSA}

Hepatic angiography was performed for the purpose of localized therapeutic planning and/or treatment with bland, chemo-, or radioembolization. Prior to each hepatic angiogram, the interventional radiologist reviewed cross sectional imaging and evaluated the location of HCC tumor(s). Hepatic angiograms were performed by one of four interventional radiologists with experience levels ranging from 11 to 30 years, using standard fluoroscopic DSA techniques and varying parameters. The majority of the studies were performed with dual plane flat panel digital angiography (Infinix DP-I; Toshiba, Tustin, CA, USA). Images were acquired during selective injection of nonionic iodinated contrast (Iopamidol $^{\circledR}$, Isovue 300, Bracco Diagnostics Inc., Monroe Township, NJ, USA) into celiac axis and/or common hepatic artery through coaxial microcatheters introduced via femoral artery sheath. In selected patients, super-selective DSA was also performed in branching hepatic arteries for improved visualization of suspicious lesions or focal treatment. Injection of contrast was performed at varying rates and volumes by manual and/or power injection (Mark V ProVis Angiographic Injection System; Medrad, Warrendale, PA, USA). C-arm CT examination images were excluded from review.

\section{Pathology}

Pathology reports of the explanted livers were used as the ref- erence standard for confirmation of $\mathrm{HCC}(\mathrm{s})$. Liver explants were examined in whole and sectioned at 3-5 mm intervals in axial plane per standard surgical pathologic protocol. Nodules appearing grossly suspicious or measuring greater than $10 \mathrm{~mm}$ are evaluated by microscopic and histologic analysis with hemotoxylin and eosin staining. All pathology reports reviewed were approved by staff pathologists, most of which were examined by one of two experienced, dedicated hepatopathologists. Pathology reports were retrospectively reviewed, and the following data was extracted: total number of tumors, size and location of each tumor, and additional lesion characteristics (including presence of embolization material). Notation of focal embolization material and underlying malignant cellular change was considered as tumor. The reported pathologic results were compared to retrospective imaging review results on a tumor-by-tumor basis according to location, size and additional descriptors.

\section{Image review}

MDCT, MRI and hepatic angiography images were grouped by modality and anonymized and randomized for review. Two fellowship trained abdominal radiologists with 11 and 5 years of experience blindly reviewed the cross-sectional studies. American Association for the Study of Liver Diseases (AASLD) imaging criteria (arterial hypervascularity and venous and/or delayed phase washout) were used for identification of HCC. ${ }^{17}$ If multiple cross sectional exams of the same modality were performed prior to transplantation, only exams performed closest to the date of transplant were included in the study. No comparison imaging or pathology reports were available during the review.

Two interventional radiologists with 11 and 18 years of experience and special interest in interventional oncology reviewed the hepatic angiograms. Again, these studies were anonymized and randomized for independent review without comparison imaging. Only angiograms performed closest to the date of transplant were included in the study. Both sets of reviewers analyzed each imaging study for the total number, size, and location of each suspected tumor (lobe and, when possible, Couinaud segment), and any additional pertinent descriptors (such as presence of suspected satellite lesions, nearby anatomic landmarks, etc). Specifically, the location of the lesions (identified as hypervascular masses) was determined by the arterial branching pattern from non-selective and selective angiogram image sets. 


\section{Statistical analysis}

Cohen's kappa coefficient was used to determine inter-observer variability. A generalized linear mixed model was used to determine sensitivity of each test overall, as well as after separating tumors by size $(<2 \mathrm{~cm}$ versus $\geq 2 \mathrm{~cm}$ ) and by location (right versus left lobe). Differences in sensitivity were considered significant if $P<0.05$. Statistical analysis for specificity of each modality was unable given the inability to produce a "true negative" value during tumor detection.

\section{RESULTS}

\section{Patients and lesions}

Among the 41 patients, 33 were men and 8 were women, with a mean age of 58 years (range: 29.4 to 72.1 years). According to pathologic analysis, a total of 116 HCCs were detected, with an average of 3 tumors per patient (range: 1 to 11 tumors). Location of tumor varied from the right lobe in 79 , the left lobe in 34 , the caudate lobe in 2, and an unspecified lobe (hilum) in one. The mean tumor size was $2.2 \mathrm{~cm}$ (range: 0.4-7.5 cm). The number of patients with small HCCs ( $<2 \mathrm{~cm}$ in size) was similar to that of patients with larger ( $\geq 2 \mathrm{~cm}$ ) ones ( $n=61$ and 55 , respectively). The mean time interval between imaging and transplant, was 5.4 months (range: 0.3-11.4 months) for MDCT, 6.3 months (range: 0.2-17.1 months) for MRI, and 5.0 months (range: 0.1-20.5 months) for hepatic angiography. Inter-observer agreement was

Table 1. Overall sensitivity of HCC detection for each imaging modality

\begin{tabular}{lcc}
\hline Modality & Sensitivity & $\mathbf{9 5 \%} \mathrm{Cl}$ \\
\hline MDCT & 49.8 & $43.7-55.9$ \\
MRI & 51.5 & $44.6-58.4$ \\
Angiography & 41.7 & $36.2-47.3$ \\
\hline
\end{tabular}

HCC, hepatocellular carcinoma; $\mathrm{Cl}$, confidence interval; $\mathrm{MDCT}$, multi-detector computed tomography; MRI, magnetic resonance imaging. excellent for DSA and very good for MDCT and MRI (Cohen's kappa coefficients of $0.83,0.73$, and 0.78 , respectively).

\section{Overall sensitivity}

The sensitivity of tumor detection was calculated for each modality using a generalized linear mixed model, fit with the exam type as a fixed effect and the patients and lesions as random effects. The outcome variable was lesion detection (identified as "yes" or "no"). The overall sensitivity of tumor detection for each modality, independent of tumor size or location, was $49.8 \%$ for MDCT, $51.5 \%$ for MRI, and 41.7\% for hepatic angiography as listed in Table 1.

When comparing sensitivities of each modality in a pair-wise fashion, there was no statistically significant difference in sensitivity between MDCT and MRI $(P=0.722)$. However, there was statistically significant difference between the sensitivity of MDCT and angiography $(P=0.05)$, and between $M R I$ and angiography $(P=0.03)$ for HCC detection.

\section{Sensitivity by size}

The tumors were then divided into two categories based on size being greater or smaller than $2 \mathrm{~cm}$. The sensitivity of each modality according to tumor size is listed in Table 2. Tumor detection was significantly greater by all modalities in identifying tumors $\geq$ $2 \mathrm{~cm}(P<0.0001)$. The pair-wise comparison of sensitivities for small tumor detection demonstrated no significant difference between modalities (MDCT vs. MRI, $P=0.857$; MDCT vs. angiography, $P=0.065$; MRI vs. angiography, $P=0.123)$. Similarly, the pairwise comparison for detection sensitivity of tumors $\geq 2 \mathrm{~cm}$ also demonstrated no significant difference between modalities (MDCT vs. MRI, $P=0.298 ; \mathrm{MDCT}$ vs. angiography, $P=0.423$; MRI vs. angiography, $P=0.069$ ).

\section{Sensitivity by lobe}

The tumors were also separated into two groups based on

Table 2. Sensitivity of HCC detection by imaging modality according to tumor size.

\begin{tabular}{|c|c|c|c|c|}
\hline \multirow{2}{*}{ Modality } & \multicolumn{2}{|c|}{$\mathrm{HCC}(<2 \mathrm{~cm})$} & \multicolumn{2}{|c|}{$\mathrm{HCC}(\geq 2 \mathrm{~cm})$} \\
\hline & Sensitivity & $95 \% \mathrm{Cl}$ & Sensitivity & $95 \% \mathrm{Cl}$ \\
\hline MDCT & 33.8 & $25.8-41.8$ & 66.9 & 58.7-75.2 \\
\hline$M R I$ & 32.7 & $23.9-41.6$ & 73.4 & 64.4-82.4 \\
\hline Angiography & 24.1 & $17.5-30.6$ & 62.2 & $54.2-70.2$ \\
\hline
\end{tabular}

HCC, hepatocellular carcinoma; $\mathrm{Cl}$, confidence interval; MDCT, multi-detector computed tomography; MRI, magnetic resonance imaging. 
which lobe they were located in. The sensitivity of each modality in relation to tumor location is listed in Table 3. The sensitivity of tumor detection was significantly greater by all modalities in identifying tumors in the right lobe compared to those in the left $(P=0.0003)$.

\section{Sensitivity by lobe and size}

When comparing the sensitivity of tumor detection by each modality according to location and size $(<2 \mathrm{~cm}$ vs. $\geq 2 \mathrm{~cm}$ ), there were significant differences. All modalities had greater sensitivity for identifying right lobe tumors $(P=0.035)$ and tumors $\geq 2 \mathrm{~cm}$ $(P<0.0001)$. Further, when accounting for both size and location (potential confounding variables), a statistically significant difference remained between imaging modalities $(P=0.043)$. Pair-wise comparison accounting for size and location demonstrated a borderline difference in the sensitivity between MDCT and angiography $(P=0.05)$ and a significant difference between MRI and angiography $(P=0.02)$. No statistical significance was found between sensitivity of MDCT and MRI $(P=0.64)$ for detecting HCCS.

\section{False positives}

False positive tumors (those suspected to be HCC by imaging, but not present on pathology) were identified for each imaging modality. Twenty-nine percent of patients evaluated by angiography had at least one false-positive lesion, whereas $29 \%$ of patients evaluated by MDCT and $22 \%$ of patients evaluated by MR had at least one false-positive lesion. False-positivite rates of three imaging modalities were not significantly different $(P=0.67)$.

\section{DISCUSSION}

Liver transplantation has become a revolutionary, curative treatment for HCC. However, cost and resource limitation make it imperative to select the most appropriate graft recipients ac- cording to evidence-based medicine and outcomes data. Despite the validated use of MDCT and MRI to evaluate and plan treatments for patients with $\mathrm{HCC}_{1}^{8-11,13,18,19}$ discordant results may be discovered during angiography, resulting in unplanned treatment changes (Fig. 1).

There is a wide range of sensitivities reported in the literature for detection of HCC on MDCT, MRI, and angiography. Studies comparing individual cross sectional imaging modalities to explant pathology have demonstrated a range of sensitivities: $50-75 \%$ for $\mathrm{MDCT}^{20-22}$ and $70-87 \%$ for MR. ${ }^{21-23}$ Angiography, albeit less well documented, is shown to have a diagnostic accuracy ranging from 33 to $77 \%{ }^{24-27}$ with drastically lower sensitivity (18\%) for lesions $\leq 2 \mathrm{~cm} .{ }^{25}$ Those studies whose sensitivities were greatest had the common methodology of communication of pre-transplant imaging findings between the radiologist and pathologist at the time of pathologic sectioning and evaluation, ${ }^{21,23,24}$ possibly leading to reference standard bias towards the imaging findings. Our study results correspond to the lower end of these spectrums for each imaging modality (49.8\% for MDCT, $51.5 \%$ for MR, and $41.7 \%$ for angiography). This may be due to lack of histologic bias and inclusion of lesions smaller than $2 \mathrm{~cm}$. As widely discussed throughout the literature, there is a much greater sensitivity for detection of larger HCC tumors, ${ }^{15,20-25}$ specifically those $>2 \mathrm{~cm}$. This is intuitive given the possible obscuration or less typical imaging characteristics of smaller lesions, including volume averaging, decreased hepatic arterial vascularization or confounding appearance of the underlying hepatic parenchyma. The results of our study also demonstrated this correlation between improved sensitivity for tumors $\geq 2 \mathrm{~cm}(66.9 \%$ vs. $33.8 \%$ for MDCT and $73.4 \%$ vs. $32.7 \%$ for MRI, and $62.2 \%$ vs. $24.1 \%$ for angiography). Interestingly, there was a statistically greater sensitivity in detection of tumors in the right hepatic lobe versus the left hepatic lobe regardless of size or imaging modality ( $55.0 \%$ vs. $42.0 \%$ for MDCT, $56.1 \%$ vs. $43.1 \%$ for MRI, and $46.9 \%$ vs. $33.9 \%$ for angiography). These findings have not previously been reported in the literature and

Table 3. Sensitivity of HCC detection by imaging modality according to tumor location.

\begin{tabular}{lccccc}
\hline \multirow{2}{*}{ Modality } & \multicolumn{3}{c}{ Right lobe } & & \multicolumn{2}{c}{ Left lobe } \\
\cline { 2 - 3 } \cline { 5 - 6 } & Sensitivity & $\mathbf{9 5 \% ~ C l}$ & & Sensitivity & \multicolumn{9}{c}{$\mathbf{9 5 \%} \mathrm{Cl}$} \\
\hline MDCT & 55.0 & $48.3-61.6$ & 42.0 & $34.2-49.8$ \\
MRI & 56.1 & $48.8-63.4$ & & 43.1 & $34.5-51.7$ \\
Angiography & 46.9 & $40.7-53.1$ & & 33.9 & $26.6-41.3$ \\
\hline
\end{tabular}

HCC, hepatocellular carcinoma; $\mathrm{Cl}$, confidence interval; MDCT, multi-detector computed tomography; MRI, magnetic resonance imaging. 
Lauren M. Ladd, et al. Comparison of Hepatic MDCT, MRI, and DSA to explant pathology
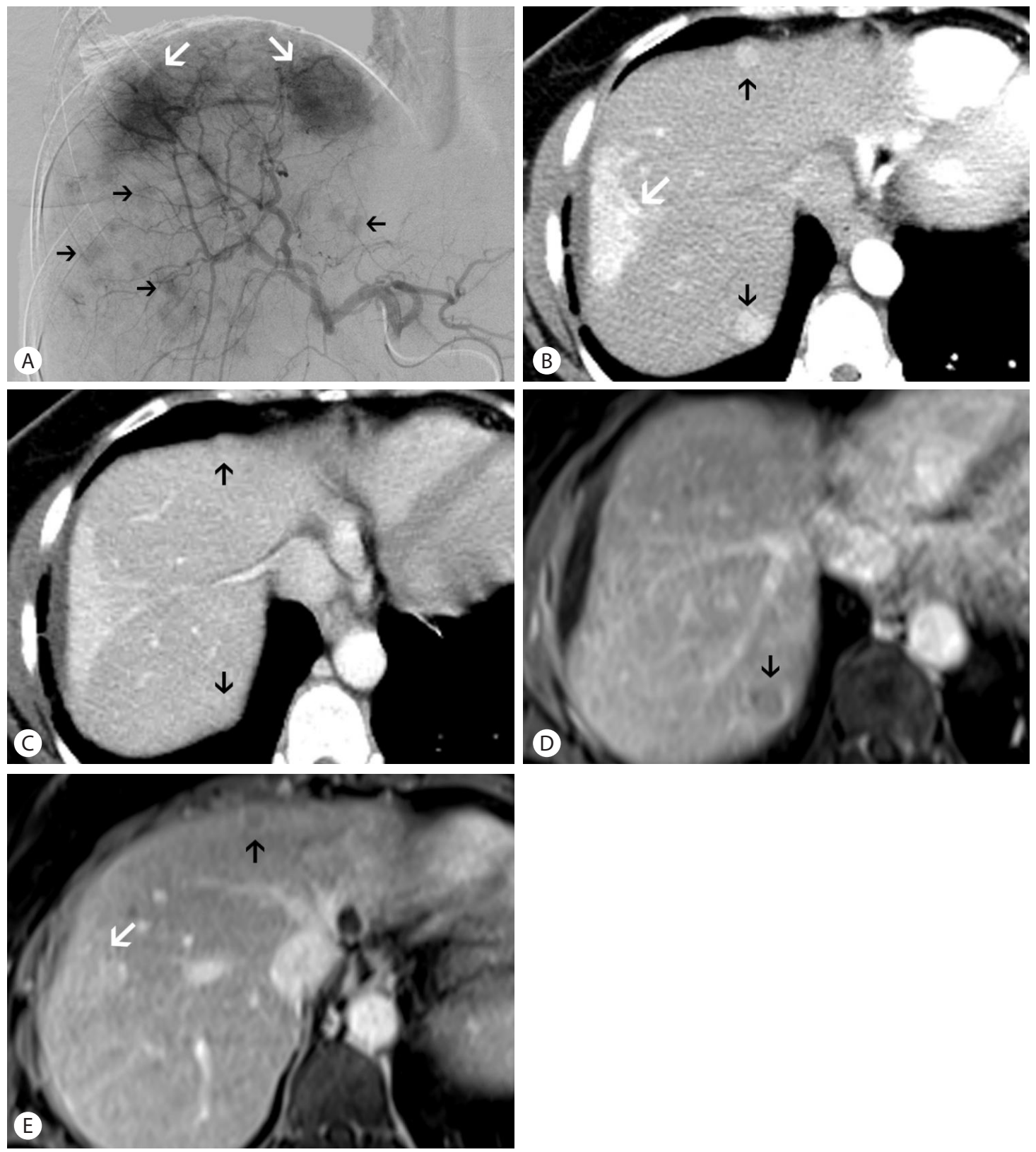

Figure 1. Imaging assessments in a 47-year-old female with cirrhosis and three histologically proven HCCs: (A) DSA, (B) arterial-phase MDCT, (C) portalvenous-phase MDCT, and (D, E) 5-minute delayed-phase MRI of the liver. (A) DSA image of the right hepatic lobe after subselective right hepatic artery injection, demonstrating two round contrast blushes (white arrows). The medial arrow corresponds to an accurately identified HCC, and the lateral arrow corresponds to a region of posttreatment change in the prior external beam radiation. Numerous additional small round hypervascular blushes (black arrows) were misidentified as multifocal HCCs. (B) Arterial-phase MDCT axial image of the liver, demonstrating round hypervascular lesions in the posteromedial right hepatic lobe and an anterior left medial hepatic lobe (black arrows) that were correctly identified as HCCs. The additional geographic enhancement in the lateral right hepatic lobe (white arrow) was hyperemia due to prior external beam radiation therapy for a previously identified HCC. No additional lesions were identified corresponding to suspicious multifocal lesions identified by DSA. (C) Portal-venous-phase MDCT axial image of the right hepatic lobe at the same level as Fig. 1B, demonstrating isoenhancement in the areas of histologically proven HCCs (black arrows). There was no washout during the portal venous phase, and diagnoses of HCCs were based on arterial hyperenhancement in a cirrhotic liver. (D) Five-minute delayedphase postcontrast T1-weighted MRI image demonstrating characteristic washout in the posteromedial right hepatic lobe (black arrow). This accurately identified tumor corresponds to the right hepatic lobe lesion identified on DSA and MDCT. (E) Five-minute delayed-phase postcontrast T1-weighted MRI image demonstrating characteristic washout in the anterior left medial hepatic lobe (black arrow), accurately identified as HCC. There is again mild persistent enhancement of the lateral right hepatic lobe at the site of prior external beam radiation. Again, no additional HCCs were identified corresponding to the suspicious multifocal disease seen on DSA. The white arrow in $1 \mathrm{E}$ denotes the region of postradiation change, which does not demonstrate washout. HCC, hepatocellular carcinoma; DSA, digital subtraction angiography; MDCT, multidetector computed tomography. 
could possibly be explained by streamlining of blood flow to the right hepatic lobe. ${ }^{28,29}$

Unique to the aforementioned literature, our study directly compares the sensitivities of MDCT, MRI and angiography within the same patient population. While comparing the results of each modality in a pair-wise fashion, we found a statistically significant difference between MRI and angiography $(P=0.03)$, which is an addition to the previous studies reporting superiority of MRI over $\mathrm{CT}^{21,30,31}$ Similarly, once adjusted for differences in lesion size and location, pair-wise comparison demonstrated the greatest and most statistically significant difference between MRI and angiography sensitivities $(P=0.02)$, as well as a borderline significance in the difference between MDCT and angiography $(P=0.05)$. These findings suggest that angiography alone should not be considered an equivalent imaging method used to affect or change the treatment pathway of these patients.

We also assessed the number of false positive lesions as another measure of comparing modality-based accuracy. False positive lesions have been evaluated in prior modality-specific studies of HCC detection. ${ }^{15,23,32}$ These studies demonstrate up to $8 \%$ false positive lesions for $\mathrm{MDCT}_{1}{ }^{23} 31 \%$ for $\mathrm{MR}_{1}{ }^{32}$ and $48 \%$ for C-arm CT angiography. ${ }^{15}$ However, none of these studies compares the false positive lesions detected by each of these modalities in the same patients. Our study compared the false positive lesions detected in each patient to explant pathology and revealed no statistically significant difference between these modalities (angiography; 29\% of patients [ $n=12$, MDCT; $29 \%$ of patients [ $n=12$ ] and MRI; $22 \%$ of patients $[n=9]$ ).

The limitations of our study include the retrospective design and variable time elapsed between each imaging modality and from imaging to transplant. The exclusion criteria for our study did not address time elapsed between imaging exams or between imaging and transplant, and a more selective group of patients would likely improve the accuracy of each modality. There were variable angiographic image acquisition techniques, largely due to exam indication (diagnostic exams for treatment planning versus exams for catheter-directed therapies) and patient cooperation. This may have led to falsely low sensitivity at time of review that would not be an accurate depiction of clinical angiography exams.

In summary, our results have demonstrated the MR and MDCT to be more accurate imaging for transplant evaluation and treatment planning. Therefore, additional angiographic findings at the time of interventional treatment should be verified with cross sectional studies before changing the patient's management. Prospective evaluation of cross sectional and interventional angiographic imaging should be pursued to optimize the evaluation of each exam's sensitivity and establish the most appropriate treatment planning pathway for liver transplant candidates with HCC.

\section{Conflicts of Interest}

Kumar Sandrasegaran is a research consultant for and has research grants from Repligen Corporation. Matthew Johnson is a research consultant for and has research grants from Boston Scientific Corporation and Rex Medical, LP.

The other authors have no financial interest or other relationship with any commercial organizations.

\section{REFERENCES}

1. Patel M, Shariff MI, Ladep NG, Thillainayagam AV, Thomas HC, Khan SA, et al. Hepatocellular carcinoma: diagnostics and screening. J Eval Clin Pract 2012;18:335-342.

2. Saar B, Kellner-Weldon F. Radiological diagnosis of hepatocellular carcinoma. Liver Int 2008;28:189-199.

3. Siegel R, Jemal A. Cancer Facts \& Figures 2012. In: American Cancer Society, 2012:1-64.

4. Centers for Disease Control and Prevention (CDC). Hepatocellular carcinoma - United States, 2001-2006. MMWR Morb Mortal Wkly Rep 2010;59:517-520.

5. Altekruse SF, McGlynn KA, Reichman ME. Hepatocellular carcinoma incidence, mortality, and survival trends in the United States from 1975 to 2005. J Clin Oncol 2009:27:1485-1491.

6. Altekruse SF, McGlynn KA, Dickie LA, Kleiner DE. Hepatocellular carcinoma confirmation, treatment, and survival in surveillance, epidemiology, and end results registries, 1992-2008. Hepatology 2012;55:476-482.

7. Hauboldt RH, Hanson SG, Bemstein GR. 2008 U. S. organ and tissue transplant cost estimates and discussion. In: Milliman, Inc., 2008:120.

8. Benson AB 3rd, Abrams TA, Ben-Josef E, Bloomston PM, Botha JF, Clary BM, et al. NCCN clinical practice guidelines in oncology: hepatobiliary cancers. J Natl Compr Canc Netw 2009;7:350-391.

9. Bruix J, Sherman M. Management of hepatocellular carcinoma. Hepatology 2005;42:1208-1236.

10. McCaughan GW, Koorey DJ, Strasser SI. Hepatocellular carcinoma: current approaches to diagnosis and management. Intern Med J 2002;32:394-400. 
11. Murray KF, Carithers RL Jr. AASLD practice guidelines: Evaluation of the patient for liver transplantation. Hepatology 2005;41:1407-1432.

12. Mazzaferro V, Regalia E, Doci R, Andreola S, Pulvirenti A, Bozzetti F, et al. Liver transplantation for the treatment of small hepatocellular carcinomas in patients with cirrhosis. N Engl J Med 1996;334:693-699.

13. Yao FY, Ferrell L, Bass NM, Bacchetti P, Ascher NL, Roberts JP. Liver transplantation for hepatocellular carcinoma: comparison of the proposed UCSF criteria with the Milan criteria and the Pittsburgh modified TNM criteria. Liver Transpl 2002;8:765-774.

14. Fowler KJ, Karimova EJ, Arauz AR, Saad NE, Brunt EM, Chapman $W C$, et al. Validation of organ procurement and transplant network (OPTN)/united network for organ sharing (UNOS) criteria for imaging diagnosis of hepatocellular carcinoma. Transplantation 2013;95:1506-1511.

15. Iwazawa J, Ohue S, Hashimoto N, Abe H, Hamuro M, Mitani T. Detection of hepatocellular carcinoma: comparison of angiographic Carm CT and MDCT. AJR Am J Roentgenol 2010;195:882-887.

16. Bruix J, Sherman M, Llovet JM, Beaugrand M, Lencioni R, Burroughs AK, et al. Clinical Management of Hepatocellular Carcinoma: Conclusions of the Barcelona-2000 EASL Conference. J Hepatol 2001;35:421-430.

17. Bruix J, Sherman M. Management of hepatocellular carcinoma: an update. Hepatology 2011;53:1020-1022.

18. Mazzaferro V, Bhoori S, Sposito C, Bongini M, Langer M, Miceli R, et al. Milan criteria in liver transplantation for hepatocellular carcinoma: an evidence-based analysis of 15 years of experience. Liver Transpl 2011;17(Suppl 2):S44-S57.

19. Tanwar S, Khan SA, Grover VP, Gwilt C, Smith B, Brown A. Liver transplantation for hepatocellular carcinoma. World J Gastroenterol 2009;15:5511-5516.

20. Addley HC, Griffin N, Shaw AS, Mannelli L, Parker RA, Aitken S, et al. Accuracy of hepatocellular carcinoma detection on multidetector CT in a transplant liver population with explant liver correlation. Clin Radiol 2011;66:349-356.

21. Burrel M, Llovet JM, Ayuso C, Iglesias C, Sala M, Miquel $R$, et al. MRI angiography is superior to helical CT for detection of HCC prior to liver transplantation: an explant correlation. Hepatology 2003;38:1034-1042.

22. Libbrecht L, Bielen D, Verslype C, Vanbeckevoort D, Pirenne J, Nevens $F$, et al. Focal lesions in cirrhotic explant livers: pathological evaluation and accuracy of pretransplantation imaging examinations. Liver Transpl 2002;8:749-761.

23. Choi SH, Lee JM, Yu NC, Suh KS, Jang JJ, Kim SH, et al. Hepatocellular carcinoma in liver transplantation candidates: detection with gadobenate dimeglumine-enhanced MRI. AJR Am J Roentgenol 2008;191:529-536.

24. Steingruber IE, Mallouhi A, Czermak BV, Waldenberger P, Gassner $E$, Offner $F$, et al. Pretransplantation evaluation of the cirrhotic liver with explantation correlation: accuracy of CT arterioportography and digital subtraction hepatic angiography in revealing hepatocellular carcinoma. AJR Am J Roentgenol 2003;181:99-108.

25. Krinsky GA, Nguyen MT, Lee VS, Rosen RJ, Goldenberg A, Theise $N D$, et al. Dysplastic nodules and hepatocellular carcinoma: sensitivity of digital subtraction hepatic arteriography with whole liver explant correlation. J Comput Assist Tomogr 2000;24:628-634.

26. Bartolozzi C, Lencioni R, Caramella D, Palla A, Bassi AM, Di Candio G. Small hepatocellular carcinoma. Detection with US, CT, MR imaging, DSA, and Lipiodol-CT. Acta Radiol 1996;37:69-74.

27. El-Gazzaz G, Sourianarayanane A, Menon KV, Sanabria J, Hashimoto K, Quintini C, et al. Radiologic-histological correlation of hepatocellular carcinoma treated via pre-liver transplant locoregional therapies. Hepatobiliary Pancreat Dis Int 2013;12:34-41.

28. Gallix BP, Reinhold C, Dauzat M, Bret PM. Streamlined flow in the portal vein: demonstration with MR angiography. J Magn Reson Imaging 2002;15:603-609.

29. Kashiwagi T, Kimura K, Suematsu T, Schichiri M, Kamada T, Abe H. Heterogeneous intrahepatic distribution of blood flow in humans. Eur J Nucl Med 1981;6:545-549.

30. Colli A, Fraquelli M, Casazza G, Massironi S, Colucci A, Conte D, et al. Accuracy of ultrasonography, spiral $C T$, magnetic resonance, and alpha-fetoprotein in diagnosing hepatocellular carcinoma: a systematic review. Am J Gastroenterol 2006;101:513-523.

31. Yu NC, Chaudhari V, Raman SS, Lassman C, Tong MJ, Busuttil RW, et al. CT and MRI improve detection of hepatocellular carcinoma, compared with ultrasound alone, in patients with cirrhosis. Clin Gastroenterol Hepatol 2011;9:161-167.

32. Brancatelli G, Baron RL, Peterson MS, Marsh W. Helical CT screening for hepatocellular carcinoma in patients with cirrhosis: frequency and causes of false-positive interpretation. AJR Am J Roentgenol 2003;180:1007-1014. 